\title{
Physiological Effects of Salmon Milt Nucleoprotein on Movement, Stress Tolerance and Lifespan of $C$. elegans
}

\author{
Hiroaki Shintani $^{1}$, Tsubasa Furuhashi ${ }^{1}$, Hiroyuki Hano ${ }^{1}$, Masaji Matsunaga ${ }^{2}$, Koji Usumi $^{3}$, \\ Norimasa Shudo ${ }^{4}$, Kazuichi Sakamoto ${ }^{1 *}$ \\ ${ }^{1}$ Graduate School of Life and Environmental Sciences, University of Tsukuba, Ibaraki, Japan; ${ }^{2}$ Gene Trophology Institute, Hokkaido, \\ Japan; ${ }^{3}$ Life Science Institute Co., Ltd., Tokyo, Japan; ${ }^{4}$ Nissan Chemical Industries, Co., Ltd., Tokyo, Japan. \\ Email: "sakamoto@biol.tsukuba.ac.jp
}

Received October $4^{\text {th }}, 2011$; revised November $15^{\text {th }}, 2011$; accepted November $23^{\text {rd }}, 2011$

\begin{abstract}
In recent years, various physiological functions of salmon milt extract, which consists of nucleic acid and nucleoprotein, have been reported. The objective of this study is to analyze the physiological function and its mechanism of salmon milt extract (NG) on nematodes (C. elegans). The wild type nematode N2 strain was bred on the plate containing of NG for four days, and its body length increased depending on NG concentration. When nematodes were bred with NG for a longer period, average lifespan was increased, and survival rate was increased by up to $20 \%$. Generally, the movement of nematodes decreases with longer breeding period (i.e. aging). Analysis of movement (both gross thrashing movement and local pumping movement) showed that NG suppressed this decrease $\mathrm{f}$ movement with aging. Furthermore, the deease of survival rate by heat stress and oxidative stress was suppressed by NG administration. Nile Red staining analysis showed that fat accumulation varied depending on the concentration of NG. RT-PCR analysis revealed that the mRNA expression levels of the stress resistance genes sod-3 and sod-4 were increased. These results indicated that NG administration increased the expression of stress-tolerance-related genes, promoted stress tolerance, increased movement and prolonged lifespan in nematode.
\end{abstract}

Keywords: Nematode; Nucleoprotein; Protamine; Lifespan; Stress; Sod

\section{Introduction}

Caenorhabditis elegans is a round worm, non-parasitic nematode and lives mainly in soil. It is easy to breed, becomes imago from fertilized eggs through four larval stages in about three days, its lifespan is short at approximately one month, and its semi-transparent body facilitates internal observation. Furthermore, its whole genome has been sequenced and so gene transfer is easy, and the species is frequently used as a human model organism because of the variety of mutants. It is also possible to constantly administer substances when culturing using $E$. coli as feed, using culture media mixed with some chemical reagents. Various experiments on the administration of substances to nematodes have been reported. For example, increase of stress tolerance by administration of green tea catechin (EGCG) [1], and prolonging of lifespan by addition of NAD [2] and resveratrol [3] have been reported. Studies using glucose overdosage to imitate the obesity model system in higher animals [4], and studies on the relation between unsaturated fatty acid or oxidative stress and physiological

${ }^{*}$ Corresponding author. function using the RNAi method $[5,6]$, have also been reported.

Salmon milt consists of nucleic acid, protein (mainly protamine) and polyamine. Generally, salmon milt is directly used as a food, and has also long been used as a food additive, such as condiment and preservative. For example, nucleic acid, which is known to show antioxidant and anti-aging effects, has long been used as an additive of powdered milk for infants. Also, amino acid arginine is abundantly contained in protamine, a major ingredient of salmon milt protein, and is known to show various physiological functions such as enhancement of immune response and metabolism, increase of fertility, and activation of insulin secretion [7-9]. Salmon milt extract contains a combination of nucleic acid and nucleoprotein, and is therefore thought to show more effecttive physiological effects. Indeed, effects such as reducetion of tumor, suppression of skin disorder by UV irradiation and improvement of exercise stamina by oral ingestion of salmon milt extract in mouse have been reported [10]. However, although various physiological functions of salmon milt extract are known, their detailed mechanism 
remains unclear. In this study, we analyzed the physiological functions of salmon milt extract for nematodes and the mechanisms of the functions using the nematode C. elegans as a model organism.

\section{Materials and Methods}

\subsection{Materials}

Nucleoprotein prepared from salmon milt was treated by nuclease and protease and was mixed into agar medium for nematode (Nissei Bio Co., Ltd., Hokkaido, Japan).

\subsection{Worms}

Wild-type Caenorhabditis elegans Bristol N2 (Caenorhabditis Genetics Center, Minnesota, USA) were cultured at $20^{\circ} \mathrm{C}$ on NGM (Nematode Growth Medium) agar plated with E. coli OP50 as previously described [11]. Each worm was transferred on the new plate in each 4 days.

\subsection{Synchronization of the Growth Stage}

To synchronize the growth stage of nematode, 300 adult worms were treated with $10 \% \mathrm{NaClO}$ solution $(10 \mathrm{~N}$ $\mathrm{NaOH} / \mathrm{NaClO}(5: 1))$ at room temperature, until the skin of the individual of $80 \%$ was destroyed. The eggs were rinsed by $\mathrm{S}$-basal $(0.1 \mathrm{~mol} / 1 \mathrm{NaCl}, 50 \mathrm{mmol} / 1$ potassium phosphate buffer), and the above $\mathrm{NaClO}$ treatment was repeated in triplicate. Finally, the collected eggs were cultured overnight in S-basal at $20^{\circ} \mathrm{C}$ until the hatching.

\subsection{Culture of Worms on Plate Containing NG}

NGM plate containing water-soluble salmon milt nuclegen (NG) was prepared for the assay. Escherichia coli strain HT115 transfected with RNAi plasmid L4440 (provided from Fire laboratory) were cultured and were plated on NGM agar containing NG, and were used for culture of nematode. A series of experiments was performed at $20^{\circ} \mathrm{C}$ except heat stress tolerance assay.

\subsection{Body Size Analysis}

The age-synchronized worms were bred on plate containing NG for $72 \mathrm{hr}$ and $96 \mathrm{hr}$. After the worms had been fixed with PFA (paraformaldehyde, Kanto Chemical, Tokyo, Japan) solution $(0.2 \%$ PFA/10\% EtOH/ $\mathrm{S}$-basal) for $5 \mathrm{~min}$, they were photographed under a microscope (DMRXA, Leica Microsystems, Inc., Bannockburn, IL, USA), and their individual lengths were measured using image analysis software Lia32 (Dr. Kazukiyo Yamamoto, Nagoya University).

\subsection{Egg Laying Analysis}

The age-synchronized worms were bred on plate con- taining NG for $72 \mathrm{hr}$, and were moved to new NGM plate. The number of eggs laid by each worm was counted over a $6 \mathrm{hr}$ period.

\subsection{Lifespan Analysis [12]}

The age-synchronized worms (20 worms/plate) were bred on plate containing $\mathrm{NG}(0,1,5,10 \mathrm{mg} / \mathrm{ml})$ plate till they grow up to adult (4th day), then worms were moved to new NG plat. The day when adult was moved was counted as day 0 , and the living population was counted every 2 days. Life or death of worms was judged by its response to the dropped S-basal. Worms were moved to new plate every 4 days to prevent the over-growth of E. coli. On day 4, 6 and 8 after the start of culture, worms were treated with $0.5 \mathrm{mg} / \mathrm{ml}$ fluorodeoxyuridine (FUdR, Wako, Osaka, Japan) to prevent the next genetical birth.

\subsection{The Movement Analysis}

The age-synchronized worms were bred plate containing $\mathrm{NG}$ for $96 \mathrm{hr}$ (day 0). Then worms were further cultured on plate containing NG for up to 9 days (0, 3, 6, 9 days). Twelve worms were randomly picked and dropped in 20 $\mu \mathrm{l}$ of S-basal on the new plate. The number of movements (pharyngeal pumping and thrashing) per $1 \mathrm{~min}$ was counted under the microscope. Worms were moved to new NG plate containing $0.5 \mathrm{mg} / \mathrm{ml} \mathrm{FUdR} \mathrm{(Wako)}$ every 4 days to prevent the over-growth of E. coli.

\subsection{Heart Stress Tolerance Assay}

The age-synchronized worms were bred plate containing NG for $72 \mathrm{hr}$. Worms were washed with S-basal buffer, transferred to bacteria-free NGM plates, and incubated at $35^{\circ} \mathrm{C}$ for $13 \mathrm{~h}$. After incubation, the worms were immediately cooled at $10^{\circ} \mathrm{C}$ for $30 \mathrm{~min}$, and the survival rate of the worms was calculated. Life or death of worms was judged by its response to the dropped S-basal. The worms which showed the viviparous hatching were eliminated.

\subsection{Oxidative Stress Tolerance Assay}

The age-synchronized worms were bred on plate containing NG for $96 \mathrm{hr}$, and the worms were transferred to new plates containing $\mathrm{NG}$ and $10 \mathrm{mM}$ paraquat $(1,1$ 'Dimethyl-4,4'-bipyridinium Dichloride (TCI Organic Chemicals, Tokyo, Japan)) (day 0). The survival rate was calculated after 6 days. Life or death of worms was judged by its response to dropped S-basal. The worms which showed the viviparous hatching were eliminated.

\subsection{Nile Red Assay}

The age-synchronized worms were bred on NGM plate 
containing $50 \mathrm{ng} / \mathrm{ml}$ Nile Red (MP Biomedicals, CA, USA) with E. Coli. After $72 \mathrm{hr}$ ' culture, worms were collected, treated with $0.2 \%$ PFA solution, and observed under a fluorescence microscope (DMRXA, Leica) with N3 filter (565 nm, Leica) [13].

\subsection{RT-PCR and Real Time PCR}

The age-synchronized worms (400 worms) were bred plate containing NG for $72 \mathrm{hr}$, and RNA was extracted by the acid-GTC-phenol method [14]. Genomic DNA was digested by treatment with DNase I (Takara, Otsu, Japan) for $60 \mathrm{~min}$ at $37^{\circ} \mathrm{C}$, and RNA was repurified by repeating the acid-GTC-phenol extraction. cDNA was synthesized by using M-MLV Reverse Transcriptase (Takara) and subjected for PCR. The quantity of cDNA was corrected by using gpd-1 (glyceraldehyde 3-phosphate dehydrogenase) as an internal standard. PCR $\left(95^{\circ} \mathrm{C}\right.$ for $5 \mathrm{~min} ; 23$ - 35 cycles of $95^{\circ} \mathrm{C}$ for $1 \mathrm{~min}, 52^{\circ} \mathrm{C}$ for $30 \mathrm{sec}, 72^{\circ} \mathrm{C}$ for $1.5 \mathrm{~min} ; 72^{\circ} \mathrm{C}$ for $7 \mathrm{~min}$ ) was performed using the specific primers for sod-3 (5'-TGC TGC AAT CTA CTG CTC-3' and 5'-ATC CTG GTT TGC ACA GGT-3'), sod-4 (5'-TAA TTC TGG CTC TCT CCG-3' and 5'-GAC GGT ACC GAT AGT TCC-3'), 5'-CAT GGA TCC ATC CAG ATG CAA AGC CAG-3' and 5'-CAT GGA TCC GTA TGC TGT GCA GCT ACA-3'), gpd-1 (5'-ATG TCG AAG GCC AAC GTC-3' and 5'-TCG CCA GTG GTG CCA GAC-3'). Real time PCR was performed using the primer for daf-16 (5'-ATC ATC TTT CCG TCC CCG-3' and 5'-TTG GAA TTG CTG GAA CCG-3'), sod-3 (5'-TAT TAA GCG CGA CTT CGG-3' and 5'-CTG GTT TGC ACA GGT GGC-3').

\subsection{Statistical Analysis}

Results are expressed as mean \pm standard deviation. Comparisons between groups were made by analysis of variance (ANOVA), and when significant, were examined by Tukey's all-pairwise-comparison test. Differences were considered significant when $P<0.05$.

\section{Results}

\subsection{NG Affected to Body Size and Reproduction}

First, body length, number of laying eggs and lifespan were measured in order to determine whether NG influences the survival rate of nematode. Eggs were treated with $\mathrm{NaClO}$ to synchronize their ages, and nematodes at the same developmental stage were used. L1 larval nematodes were bred on a plate containing various concentrations of salmon milt extract (NG), and the body length of the nematodes increased at both $72 \mathrm{hr}$ and $96 \mathrm{hr}$ (Figure 1). Especially, after $72 \mathrm{hr}$ incubation, increase of the body length, depending on the concentration of NG, was observed. The age-synchronized L1 larva was bred on an
NG plate for $72 \mathrm{hr}$, and the obtained young imago (young adult) was used to measure the number of laid eggs every $6 \mathrm{hr}$ (Figure 2). The number of laid eggs was hardly changed by the addition of NG.

\subsection{NG Prolonged the Lifespan of Nematode}

Furthermore, the age-synchronized L1 larvae were bred on an NG plate for $96 \mathrm{hr}$ (day 0 ), then the number of living individuals was measured every 2 days. Life and death of the nematodes was judged from reactivity (movement) after dropping of S-basal. As a result, the average lifespan (days of $50 \%$ survival rates) was increased by the influence of NG (Figure 3). Especially, with low concentration of $\mathrm{NG}(1 \mathrm{mg} / \mathrm{ml})$, the prolonging effect was most remarkable and the lifespan was increased by more than 2 days. On the other hand, at high concentrations of NG $(5,10 \mathrm{mg} / \mathrm{ml})$, no remarkable prolonging effect was observed. Concurrently, growth of E. coli, which was used as feed, was remarkably stimulated depending on the NG concentration. Generally, survival rate greatly decreased when the movement of nematodes is inhibited by the excessive E. coli. The increase of $E$. coli on the high NG concentration plate $(5,10 \mathrm{mg} / \mathrm{ml})$ was so intense that the movement of nematodes was restricted. Incidentally,

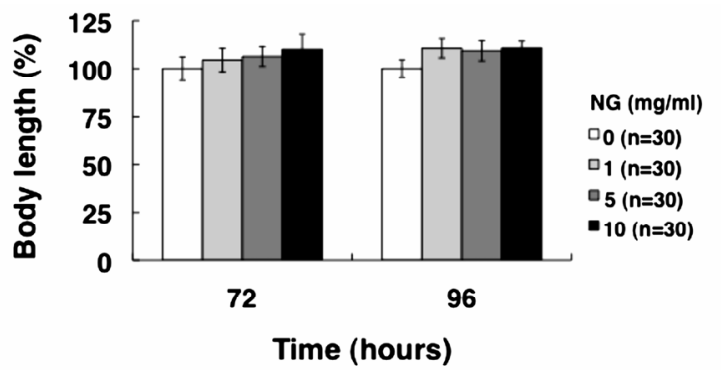

Figure 1. NG increased the body size of nematode. The synchronized L1 worms were bred on plate containing NG $(0,1$, $5,10 \mathrm{mg} / \mathrm{ml}$ ) for $72 \mathrm{hr}$ and $96 \mathrm{hr}$. Worms were fixed with PFA and their individual lengths were measured using image analysis software. $n=30$, error bar indicates standard deviation.

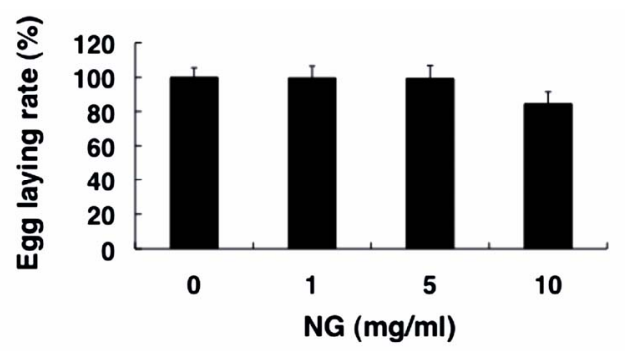

Figure 2. NG had no effect on egg laying. The synchronized L1 worms were bred on plate containing NG (0, 1, 5, 10 $\mathrm{mg} / \mathrm{ml}$ ) for $72 \mathrm{hr}$, and were moved to new NGM plate. The number of eggs laid by each worm was counted over a 6 hr period. $n=12$, error bar indicates standard deviation. 


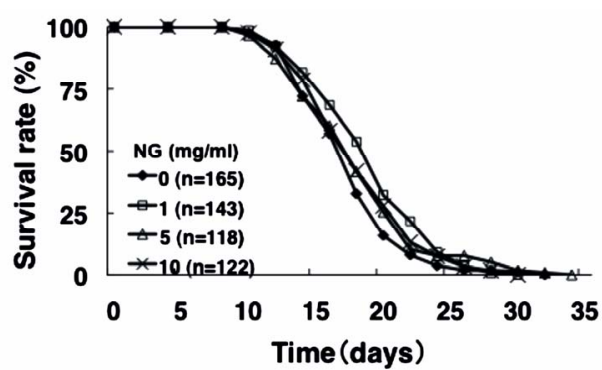

Figure 3. NG prolonged the lifespan of nematode. The synchronized $L 1$ worms ( 20 worms/plate) were bred on plate containing NG $(0,1,5,10 \mathrm{mg} / \mathrm{ml})$ for $96 \mathrm{hr}$, then worms were moved to new NG plate (day 0 ). The living population was counted every 2 days. Worms were moved to new plate every 4 days to prevent the over-growth of $E$. coli. Life or death of worms was judged by its response to the dropped S-basal. $n=165$ (NG; $0 \mathrm{mg} / \mathrm{ml}), n=143$ (NG; $1 \mathrm{mg} / \mathrm{ml}), n=$ 118 (NG; $5 \mathrm{mg} / \mathrm{ml}), n=122$ (NG; $10 \mathrm{mg} / \mathrm{ml}$ ), error bar indicates standard deviation.

FUdR was added to prevent excessive growth of E. coli in all experiments.

\subsection{NG Increased the Movement Activity of Nematode}

Generally, mature nematodes grown from parenchymula show gradually decreasing movement as aging progresses [15]. The movement is closely related to the entire lifespan, and individuals which continue to move tend to live longer. Therefore, we analyzed the physiological function of NG for the movement of nematode. Two criteria, gross movement (thrashing) and local movement of pharyngeal shrinkage (pumping), were employed as indications of movement. The age-synchronized L1 larvae were bred on an NG plate for $96 \mathrm{hr}$ (day 0), and movement per minute after a certain period of breeding $(0,3,6,9$ days $)$ was measured. As a result, the number of movements, both thrashing and pumping, decreased as the days of culture passed. On the other hand, the numbers of both thrashing and pumping movements were increased by the addition of $\mathrm{NG}$ depending on the concentration of the $\mathrm{NG}$ (Figure 4). Although no significant difference due to individual differences was observed, both local and systemic movements were increased in an NG-concentration dependent manner.

\subsection{NG Increased the Stress Tolerance of Nematode}

Next, we examined the physiological effects of NG on the stress response of nematode, using two types of stress, heat stress and oxidative stress. The optimal temperature for the survival of nematodes is around $20^{\circ} \mathrm{C}$, and the survival rate decreased to approximately $50 \%$ after exposure to the high temperature of $35^{\circ} \mathrm{C}$ for $13 \mathrm{hr}$ (Figure 5(a)).

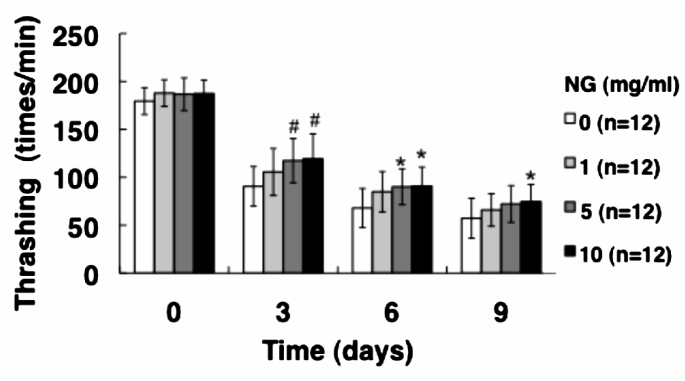

(a)

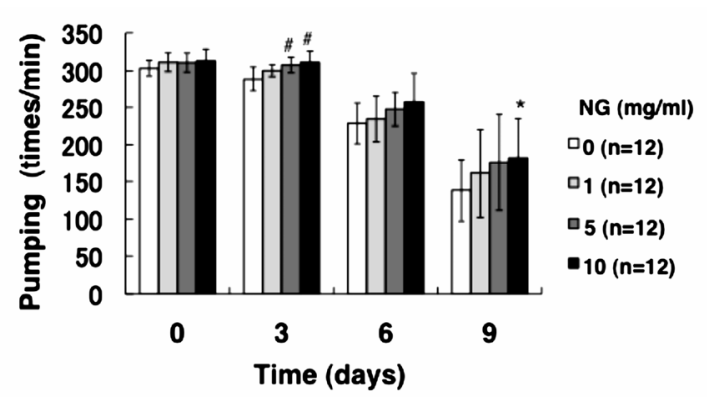

(b)

Figure 4. NG increased the movement activity of nematode. The synchronized $\mathrm{L} 1$ worms were bred on plate containing NG $(0,1,5,10 \mathrm{mg} / \mathrm{ml})$ for $96 \mathrm{hr}$ (day 0$)$. Then worms were further cultured on plate containing NG for $0,3,6,9$ days. Twelve worms were randomly picked and dropped in $20 \mathrm{ml}$ of S-basal on the new plate. The number of thrashing (a) and pharyngeal pumping (b) per $1 \mathrm{~min}$ was counted under the microscope. Worms were moved to new NG plate containing $0.5 \mathrm{mg} / \mathrm{ml}$ FUdR (Wako) every 4 days to prevent the over-growth of $E$. coli. $n=12$, error bar indicates standard deviation.

In contrast, the survival rate of nematodes bred in the presence of NG under the same heat stress increased to more than $70 \%$. Furthermore, nematodes were bred on an NG plate containing paraquat as an oxidative stress source. When paraquat is incorporated into the body, reactive oxygen species are produced. Nematodes that had been bred on the $\mathrm{NG}(+/-)$ plate $96 \mathrm{hr}$ earlier were subsequently bred on the $\mathrm{NG}(+/-)$ plate containing $10 \mathrm{mM}$ paraquat. As a result, survival rates of approximately $75 \%$ in the presence of low NG concentration $(1 \mathrm{mg} / \mathrm{ml})$ and $90 \%$ in the presence of high $\mathrm{NG}$ concentration $(5,10$ $\mathrm{mg} / \mathrm{ml}$ ) were observed, whereas the survival rate was approximately $45 \%$ in the case of NG (-) (Figure 5(b)). Therefore, $\mathrm{NG}$ was proven to remarkably increase the tolerance of nematodes to heat stress and oxidative stress.

\subsection{The Effect of NG on Fat Accumulation}

Next, we analyzed the change of adiposity by using the Nile Red method. Red fluorescence is developed by Nile Red staining when lipid droplets exist, and intense fluorescence develops as the amount of the lipid droplet 


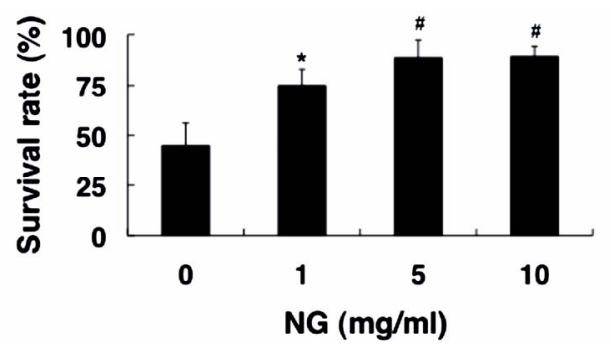

(a)

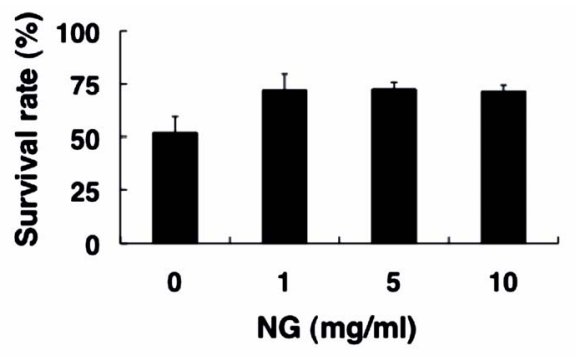

(b)

Figure 5. NG increased the stress tolerance of nematode. (a) The synchronized $L 1$ worms were bred plate containing NG $(0,1,5,10 \mathrm{mg} / \mathrm{ml})$ for $72 \mathrm{hr}$. Worms were washed with S-basal buffer, transferred to bacteria-free plates, and incubated at $35^{\circ} \mathrm{C}$ for $13 \mathrm{~h}$. After incubation, the worms were immediately cooled at $10^{\circ} \mathrm{C}$, and the survival rate was calculated; (b) The synchronized $L 1$ worms were bred on plate containing NG $(0,1,5,10 \mathrm{mg} / \mathrm{ml})$ for $96 \mathrm{hr}$, and the worms were transferred to new NG plates containing $10 \mathrm{mM}$ paraquat (day 0 ). The survival rate was calculated after 6 days. Life or death of worms was judged by its response to dropped S-basal. The worms which showed the viviparous hatching were eliminated. $n=3$, Error bar indicates standard deviation.

is increased. In the case of 1 and $5 \mathrm{mg} / \mathrm{ml} \mathrm{NG}$ concentrations, fluorescence after the 150 synchronized individuals were cultured in culture media containing Nile Red for $72 \mathrm{hr}$ was stronger than that of NG (-). On the other hand, in the presence of $10 \mathrm{mg} / \mathrm{ml} \mathrm{NG}$, fluorescence was hardly observed (Figure 6). Therefore, NG was proven to influence the amount of fat accumulation in the nematode body. However, according to recent studies, the Nile Red method does not stain triglyceride (TG) directly.

\subsection{NG Increased the Gene Expression of Sod}

Generally, daf-16, which is known as a longevity gene, is considered to be closely related to fatty acid metabolism and adiposity. A previous study showed that the expression level of daf-16 in individuals which presented intense fluorescence by Nile Red staining was increased [16]. Therefore, we analyzed gene expression by the RTPCR method. RNA was extracted from individuals bred on the NG plate for $72 \mathrm{hr}$, and the expression levels of daf-16, sod-3 and sod-4 gene were measured by the RTPCR method. As a result, the expression level of sod-3 was

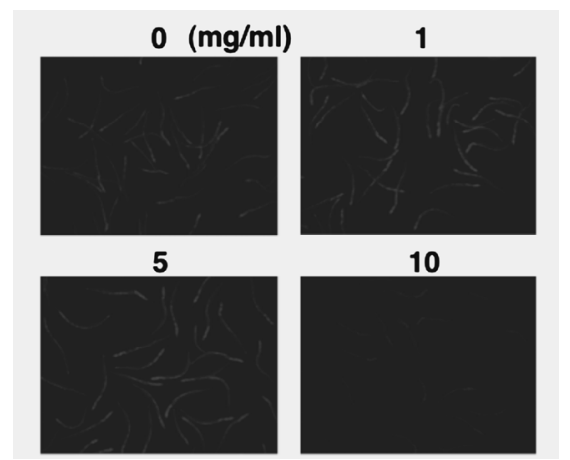

Figure 6. The effect of NG on fat accumulation. The synchronized $\mathrm{L} 1$ worms were bred on plate containing $50 \mathrm{ng} / \mathrm{ml}$ Nile Red with $E$. Coli. After 72 hr' culture, worms were collected, treated with $0.2 \%$ PFA solution, and observed under a fluorescence microscope with N3 filter.

increased after breeding on the $\mathrm{NG}(+)$ plate (Figure 7), and the expression level of sod-4 was increased by NG (1 $\mathrm{mg} / \mathrm{ml}$ ), whereas the expression of daf-16 was changed only a little. Quantitative PCR verified the similar tendency of mRNA expression of daf-16 and sod-3 (Figure 7(b)). Because the activity of transcription factor Daf-16 is affected by protein modifications such as phosphorylation and acetylation, it is currently analyzed at the protein level.

\section{Discussion}

The results of this study showed that the average lifespan (days showing 50\% survival rates) of the nematodes was prolonged by the addition of NG (Figure 3). Especially, in the case of a low concentration of $\mathrm{NG}(1 \mathrm{mg} / \mathrm{ml})$, the prolonging effect was most remarkable and the lifespan was extended by more than 2 days. Generally, nematodes bred under caloric restriction and variants with low mobility show prolonged individual lifespans [17]. Thus, the lifespan under caloric restriction is thought to be prolonged through the activation of Sir 2.1 by an increase of NAD [2]. However, the amount of feed in this study was abundant and the nematodes took sufficient calories. Considering the number of times of gross movement (thrashing), movement was restored by the addition of NG (Figure 4(a)). Further, in an experiment of pharyngeal pumping, calorie intake with $\mathrm{NG}$ supplied was higher than that of the control group (Figure 4(b)). However, the longer lifespan induced by intake of NG (Figure 3) suggests that NG affects a different pathway from that affected by caloric restriction. In addition, lifespan was not remarkably prolonged under a high concentration of $\mathrm{NG}(5,10 \mathrm{mg} / \mathrm{ml})$ (Figure 3). The reason is thought to be an inhibition of movement of nematodes due to increase of $E$. coli used for feeding after addition of $\mathrm{NG}$, leading to a decreased survival rate. The body length was increased by the addition of NG in a con- 


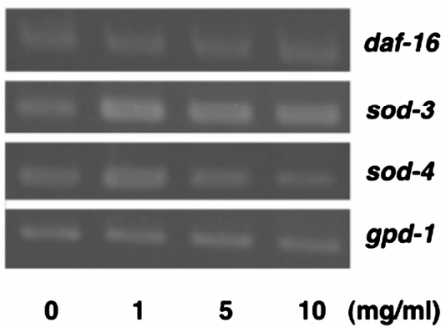

(A)

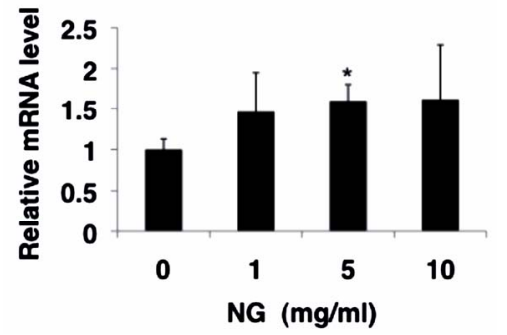

(a)

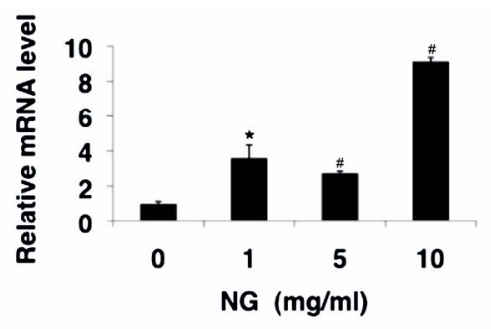

(b)

(B)

Figure 7. NG affected to gene expression. The synchronized L1 worms (400 worms) were bred plate containing NG for 72 hr, and RNA was prepared for RT-PCR. (A) The quantity of cDNA was corrected by using gpd-1 as an internal standard. PCR $\left(95^{\circ} \mathrm{C}\right.$ for $5 \mathrm{~min}$; $23-35$ cycles of $95^{\circ} \mathrm{C}$ for $1 \mathrm{~min}, 52^{\circ} \mathrm{C}$ for $30 \mathrm{sec}, 72^{\circ} \mathrm{C}$ for $1.5 \mathrm{~min} ; 72^{\circ} \mathrm{C}$ for $7 \mathrm{~min}$ ) was performed using the each primer for daf-16, sod-3 and sod-4; (B) The mRNA level of each gene was analyzed by real-time PCR. PCR (95 ${ }^{\circ} \mathrm{C}$ for 15 $\mathrm{s}, 60^{\circ} \mathrm{C}$ for $1 \mathrm{~min}$, for 40 cycles) was performed using the specific primers for daf-16 (a) and sod-3 (b).

centration-dependent manner (Figure 1). Furthermore, the number of laid eggs was not influenced by NG (Figure 2), and adiposity varied with the NG concentration (Figure 6). Generally, prolonging of lifespan and fatty acid metabolism or adiposity are known to be closely associated with transcription factor daf-16 [2,16]. Although the molecular mechanism of NG activity is unknown, the finding of this study that the amount of fat accumulation and body length vary with $\mathrm{NG}$ concentration is interesting.

In this study, both thrashing and pumping movement decreased as the breeding days passed (Figure 4). This means that movement decreased with aging of nematode. However, in the presence of NG, the number of both movements was increased depending on the addition concentration of NG (Figure 4). Therefore, it is suggested that NG is effective for restoring lost mobility with individual aging. Further, a remarkable increase of survival rate of nematodes bred in the presence of NG under heat stress is particularly interesting (Figure 5(a)). Also, although the survival rate of nematodes is greatly decreased by active oxygen stimulation, by addition of NG, the survival rate was increased (Figure 5(b)). These results indicate that the tolerance of nematodes to heat stress and oxidative stress were remarkably increased by NG.

According to gene expression analysis, the expression of sod-3 [18] and sod-4 was increased by addition of NG (Figure 7). sod-3 and sod-4 are scavenger genes involved in the removal of active oxygen, therefore the longer lifespan and greater stress tolerance induced by NG may be due to the increase of these sod gene expressions. Generally, sod-3 is controlled by transcription factor daf16 [19], which is known as a longevity gene and is related to longer lifespan [20], adiposity [16], and stress tolerance [21]. Because NG extends lifespan and promotes stress tolerance, NG may be involved to the increase of sod-3 expression and resultant reduction of ROS level through daf-16. However, RT-PCR showed that the expression of mRNA of daf-16 was hardly influenced directly by NG (Figure 7). Meanwhile, sod-4 is also a stress resistance gene, but is controlled by pha-4, not daf-16 [22]. In addition, SKN-1, a transcription factor which controls the expression of ROS-scavenging genes might be also involved in the physiological function evoked by NG [23]. The activity of these transcription factors, including daf-16, may be changed through protein modifications such as phosphorylation and acetylation, as well as changes of RNA expression level. Further detailed analysis of protein, as well as RNA level analysis, including the contribution of transcription factors other than daf-16, are necessary in future. Also, NG shows an effect of restoring movement lost with aging. The degree of influence on increasing the expression level of stress resistance genes, such as sod, on movement, as well as its detailed mechanisms, remains unclear. Factors other than sod, relating to stress tolerance, e.g. heat shock protein, may be involved. Factors relating to movement such as AMPK require a more detailed analysis.

\section{Conclusion}

In this study, we analyzed the physiological functions of salmon milt extract for nematodes and the mechanisms of the functions using the nematode C. elegans as a model organism. As the results, salmon milt extract increased average lifespan of nematode, and restored the decreased movement with aging. Furthermore, salmon milt extract promoted stress tolerance against heat and oxidative stress by upregulating the expression of stress-tolerance-related genes. These results suggested that salmon milt nucleoprotein possess multiple physiological effects in model animal, and show the potential availability as useful materials for human health.

\section{Acknowledgements}

This work was supported in part by Grants-in-Aid for 
Scientific Research from the Ministry of Education, Science, Sports, and Culture of Japan.

\section{REFERENCES}

[1] N. Saul, K. Pietsch, R. Menzel, S. R. Sturzenbaum and C. E. Steinberg, "Catechin Induced Longevity in C. elegans: From Key Regulator Genes to Disposable Soma," Mechanisms of Ageing and Development, Vol. 130, No. 8, 2009, pp. 477-486. doi:10.1016/j.mad.2009.05.005

[2] T. Hashimoto, M. Horikawa, T. Nomura and K. Sakamoto, "Nicotinamide Adenine Dinucleotide Extends the Lifespan of Caenorhabditis elegans Mediated by Sir-2.1 and Daf-16," Biogerontology, Vol. 11, No. 1, 2010, pp. 31-43. doi:10.1007/s10522-009-9225-3

[3] J. J. Collins, K. Evason and K. Kornfeld, "Pharmacology of Delayed Aging and Extended Lifespan of Caenorhabditis elegans," Experimental Gerontology, Vol. 41, No. 10, 2006, pp. 1032-9103. doi:10.1016/j.exger.2006.06.038

[4] T. Nomura, M. Horikawa, S. Shimamura, T. Hashimoto and K. Sakamoto, "Fat Accumulation in Caenorhabditis elegans is Mediated by SREBP Homolog SBP-1," Genes and Nutrition, Vol. 5, No. 1, 2010, pp. 17-27. doi:10.1007/s12263-009-0157-y

[5] M. Horikawa and K. Sakamoto, "Polyunsaturated Fatty Acids Are Involved in Regulatory Mechanism of Fatty Acid Homeostasis via Daf-2/Insulin Signaling in Caenorhabditis elegans," Molecular and Cellular Endocrinology, Vol. 323, No. 2, 2010, pp. 183-192.

doi:10.1016/j.mce.2010.03.004

[6] M. Horikawa and K. Sakamoto, "Fatty Acid Metabolism is Involved in Stress Resistance Mechanisms of Caenorhabditis elegans," Biochemical and Biophysical Research Communications, Vol. 390, No. 4, 2009, pp. 1402-1407. doi:10.1016/j.bbrc.2009.11.006

[7] A. Zajac, S. Poprzecki, A. Zebrowska, M. Chalimoniuk and J. Langfort, "Arginine and Ornithine Supplementation Increases Growth Hormone and Insulin-Like Growth Factor-1 Serum Levels after Heavy-Resistance Exercise in Strength-Trained Athletes," Journal of Strength and Conditioning Research, Vol. 24, No. 4, 2010, pp. 1082-1090. doi:10.1519/JSC.0b013e3181d321ff

[8] M. B. Witte, F. J. Thornton, U. Tantry and A. Barbuk, "L-Arginine Supplementation Enhances Diabetic Wound Healing: Involvement of the Nitric Oxide Synthase and Arginase Pathways," Metabolism, Vol. 51, No. 10, 2002, pp. 1269-1273. doi:10.1053/meta.2002.35185

[9] O. Pineiro, M. A. Ortiz, E. R. Mora, M. D. Hernandez-Navarro, R. G. Ceballos and C. G. Chamorro, "Effect of L-Arginine Oral Supplementation on Response to Myocardial Infarction in Hyper Cholesterolemic and Hypertensive Rats," Plant Foods for Human Nutrition, Vol. 65, No. 1, 2010, pp. 31-37. doi:10.1007/s11130-009-0143-y

[10] E. A. Alymkina, E. V. Dolgova, A. S. Likhacheva, V. A. Roqachev, E. S. Tamara, P. N. Valeriy, A. P. Nelly, E. O. Konstantin, N. S. Dmitriy, R. C. Elena, N. Z. Stanislav, S. B. Sergei and A. S. Mikhail, "Combined Therapy with Cyclophosphamide and DNA Preparation Inhibits The Tumor Growth in Mice," Genetic Vaccines and Therapy, Vol. 14, 2009, pp. 7-12. doi:10.1016/j.mad.2009.05.005
[11] S. Brenner, "The Genetics of Caenorhabditis Elegans," Genetics, Vol. 77, No. 1, 1974, pp. 71-94.

[12] H. Hsin and C. Kenyon, "Signals from the Reproductive System Regulate the Lifespan of C. elegans," Nature, Vol. 399, 1999, pp. 362-366.

[13] M. Horikawa, T. Nomura, T. Hashimoto and K. Sakamoto, "Elongation and Desaturation of Fatty Acids Are Critical in Growth, Lipid Metabolism, and Ontogeny of Caenorhabditis Elegans," Journal of Biochemistry, Vol. 144, No. 2, 2008, pp. 149-158. doi:10.1093/jb/mvn055

[14] P. Chomczynski and N. Sacchi, "Single-Step Method of Rna Isolation by Acid Guanidinium Thiocyanate-PhenolChloroform Extraction," Analytical Biochemistry, Vol. 162, No. 1, 1987, pp. 156-159.

[15] C. Hung, C. Xiong and K. Kornfeld, "Measurements of Age-Related Changes of Physiological Processes That Predict Lifespan of Caenorhabditis elegans," Proceedings of the National Academy of Sciences of the United States of America, Vol. 101, 2004, pp. 8084-8089. doi: 10.1073/pnas.0400848101

[16] K. Ashrafi, F. Y. Chang, J. L. Watts, G. F. Andrew, S. K. Ravi, A. Julie and R. Gary, "Genome-Wide RNAi Analysis of Caenorhabditis elegans Fat Regulatory Genes," Nature, Vol. 421, No. 6920, 2003, pp. 268-272.

[17] D. Cristina, M. Cary, A. Lunceford, C. Clarke and C. Kenyon, "A Regulated Response to Impaired Respiration Slows Behavioral Rates and Increases Lifespan in Caenorhabditis elegans," PLoS Genetics, Vol. 5, No. 4, 2009, pp. 1-15. doi:10.1371/journal.pgen.1000450

[18] M. P. Giglio, T. Hunter, J. V. Bannister and G. J. Hunter, "The Manganese Superoxide Dismutase Gene of Caenorhabditis elegans," Biochemistry \& Molecular Biology International, Vol. 33, No. 1, 1994, pp. 37-40.

[19] T. Furuyama, T. Nakazawa, I. Nakano and N. Mori, "Identification of Differential Distribution Patterns of mRNAs and Consensus Binding Sequences for Mouse DAF-16 Homologues," Biochemical Journal, Vol. 349, 2000, pp. 629-634.

[20] C. T. Murphy, S. A. McCarroll, C. I. Barqmann, A. Fraser, R. S. Kamath, S. Ahringer, H. Li and C. Kenyon, "Genes that Act Downstream of DAF-16 to Influence the Lifespan of Caenorhabditis elegans," Nature, Vol. 424, No. 6946, 2003, pp. 277-283.

[21] Y. Honda and S. Honda, "The Daf-2 Gene Network for Longevity Regulates Oxidative Stress Resistance and MnSuperoxide Dismutase Gene Expression in Caenorhabditis elegans," The Federation of American Societies for Experimental Biology Journal, Vol. 13, No. 11, 1999, pp. 1358-1393. doi:10.1038/nature05837

[22] S. H. Panowski, S. Wolff, H. Aguilaniu, J. Durieux and A. Dillin, "PHA-4/Foxa Mediates Diet-Restriction-Induced Longevity of C. elegans," Nature, Vol. 447, No. 7144, 2007, pp. 550-55.

[23] J. H. An, K. Vranas, M. Lucke, H. Inoue, N. Hisamoto, K. Matsumoto and T. K. Blackwell, "Regulation of the Caenorhabditis elegans Oxidative Stress Defense Protein SKN1 by Glycogen Synthase Kinase-3," Proceedings of the National Academy of Sciences of the United States, Vol. 102 , No. 45, 2005, pp. 16275-16280. doi: $10.1073 /$ pnas. 0508105102 